\title{
Impact of obesity on the prognosis of hypertensive disorders in pregnancy
}

\author{
ROXANA ELENA BOHILTEA ${ }^{1 *}$, CORINA-AURELIA ZUGRAVU ${ }^{2 *}$, \\ DRAGOS NEMESCU ${ }^{3 *}$, NATALIA TURCAN ${ }^{4}$, FLORINA-PAULA PAULET $^{4}$, \\ FLORENTINA GHERGHICEANU $^{5}$, IONITA DUCU ${ }^{1}$ and MONICA MIHAELA CIRSTOIU ${ }^{1}$ \\ ${ }^{1}$ Department of Obstetrics and Gynecology, 'Carol Davila' University of Medicine and Pharmacy, \\ University Emergency Hospital of Bucharest, 050098 Bucharest; ${ }^{2}$ Department of Food Hygiene and Nutrition, \\ 'Carol Davila' University of Medicine and Pharmacy, 050463 Bucharest; ${ }^{3}$ Department of Obstetrics and Gynecology, \\ 'Grigore T. Popa' University of Medicine and Pharmacy, 700115 Iasi; ${ }^{4}$ Department of Obstetrics and Gynecology, \\ 'Carol Davila' University of Medicine and Pharmacy Doctoral School, University Emergency Hospital of Bucharest, \\ 050098 Bucharest; ${ }^{5}$ Department of Marketing and Medical Technology, 'Carol Davila' University \\ of Medicine and Pharmacy, 050474 Bucharest, Romania
}

Received March 9, 2020; Accepted April 9, 2020

DOI: $10.3892 / \mathrm{etm} .2020 .8783$

\begin{abstract}
The prevalence of pathologies due to placental dysfunction superimposed on pregnancy is constantly increasing. The prognosis of the cases complicated by gestational hypertension is usually good, significantly better compared with that of the cases associating preeclampsia. About half of the cases with gestational hypertension will progress to preeclampsia, the risk of decompensation being inversely proportional to the gestational age of the onset of gestational hypertension. The present study, analyzed the cases of pregnancy and postpartum complicated by pathologies related to placental dysfunction, during a period of 5 years. The risk factors analyzed were the presence of infections during pregnancy, diabetes, thrombophilia, pregnancy obtained by in vitro fertilization, abnormal adherence of the placenta, obesity, multiple pregnancy, the presence of an earlier hepatic, endocrine, renal, cardiac or autoimmune pathology, and the existence of an uterine malformation. Obesity appears with a significantly increased incidence in patients with gestational hypertension and middle preeclampsia. Intrauterine growth restriction appears with a significantly increased incidence in patients with mild preeclampsia. Complications such as prematurity, acute fetal distress and abruption of placentae
\end{abstract}

Correspondence to: Dr Natalia Turcan, Department of Obstetrics and Gynecology, 'Carol Davila' University of Medicine and Pharmacy Doctoral School, University Emergency Hospital of Bucharest, 169 Splaiul Independenței, 050098 Bucharest, Romania

E-mail: napritcan@gmail.com

*Contributed equally

Key words: obesity, hypertension, preeclampsia, prematurity, intrauterine growth restriction had a significantly increased incidence in patients with severe preeclampsia. Thus, obese patients have a higher risk of moderate preeclampsia, following gestational hypertension and finally severe preeclampsia.

\section{Introduction}

Regarding the complications, respectively, maternal and fetal morbidity and mortality, preeclampsia remains an ongoing topic. Preeclampsia is called the disease of 'theories', given the fact that it remains an enigma from many points of view. In the last two decades, however, significant progress has been made in elucidating the pathogenesis of preeclampsia, which is the key to early detection of this condition and to the appropriate therapeutic pathway (1). Even though there are numerous studies concerning this topic, it is imperative we clarify the methods of prevention and treatment, through a well-established protocol with clear classification of the patient in the high risk group and individualization of management and monitoring according to a well-established scheme. The prevalence of pathologies due to placental dysfunction superimposed on pregnancy is constantly increasing in the context in which the maternal age is also increasing from one decade to another. However, in a significant number of cases, preeclampsia occurs in nulliparous patients, with an apparent health status that presents no obvious risk factors (2). The impact of preeclampsia is not only limited to the specific complications during pregnancy, birth or postpartum, but also continues for a long time. The risk of maternal death is significantly higher in patients who develop severe, early preeclampsia with HR, 9.54; 95\% CI, 4.50-20.25 (3).

The prognosis of the cases complicated by gestational hypertension is usually good, significantly better compared with that of the cases associating preeclampsia, but the concern about the complications that may occur should be just as great. About half of the cases with gestational hypertension 
will progress to preeclampsia, the risk of decompensation being inversely proportional to the gestational age of the onset of gestational hypertension (4). Respectively, the earlier the gestational hypertension occurs during pregnancy, the greater the risk of progression to preeclampsia (5).

\section{Subjects and methods}

The present study, analyzed the cases of pregnancy and postpartum complicated by pathologies related to placental dysfunction, during a period of 5 years (2015-2019), by collecting data from the medical statistics department of the University Emergency Hospital Bucharest. The case groups include the control group 1, respectively the general population, group 2 , patients with preexisting pregnancy hypertension, group 3 patients with gestational hypertension, group 4 patients with moderate preeclampsia, group 5 patients with severe preeclampsia, and group 6 patients with eclampsia. In the first stage of statistical processing, descriptive tests were applied and the presence of risk factors and complications in the studied groups was analyzed. In the context in which the distribution of the data was not a Gaussian one, non-parametric tests, respectively, Man Whitney U were applied. For a better visualization and interpretation of the results, classification tests were used. The program used was SPSS 12.0 and the statistical significance threshold considered was $\mathrm{P}<0.05$.

During the 5 years of study there were 67 cases of pre-existing hypertension, 240 cases of gestational hypertension, 319 cases of moderate preeclampsia, 130 cases of severe preeclampsia and 16 cases of eclampsia (Fig. 1).

The main associated complications analyzed were birth by Caesarean section, fetal death, intrauterine growth restriction, oligohydramnios, gestational edema, premature birth, preterm and premature rupture of membranes, HELLP syndrome, abruptio placentae and caesarean hysterectomy. The frequency for each studied group and their comparative reproduction is shown in Fig. 2.

The risk factors analyzed were the presence of infections during pregnancy, diabetes, thrombophilia, pregnancy obtained by in vitro fertilization, abnormal adherence of the placenta, obesity, multiple pregnancy, the presence of an earlier hepatic, endocrine, renal, cardiac or autoimmune pathology, and the existence of an uterine malformation. The frequency on the studied groups is shown comparatively in Fig. 3.

\section{Results}

According to Mann Whitney tests, significant results for the incidence of obesity were obtained by comparative analysis of control group-1 (general population) with groups 3, 4 and 5, respectively, patients with gestational hypertension, moderate preeclampsia and severe preeclampsia. Obesity appears with a significantly increased incidence in patients with gestational hypertension and middle preeclampsia. IUGR appears with a significantly increased incidence in patients with mild preeclampsia. Complications such as prematurity, acute fetal distress and abruption placentae had a significantly increased incidence in patients with severe preeclampsia. The results of the Mann Whitney tests are shown in Table I, where only the coefficients of statistically significant relationships are shown.
Following the value of $r$, medium size effect was recorded for obesity as a risk factor for mild preeclampsia, compared with the general population, and varying from the upper limit to large effect for oligohydramnios as a complication of mild preeclampsia. Thus, the results show an important statistical association and a cause-effect relationship for the conditions described above compared with the other variables. For patients with mild preeclampsia, we obtained a significantly higher incidence of obesity and a high risk of oligohydramnios.

The effect of obesity is medium compared between the two groups, respectively mild and severe preeclampsia, and significantly differentiates the risk for mild preeclampsia.

In the context in which obesity was found as a significant risk factor through non-parametric comparative tests between the studied groups, a statistical analysis was used to establish the severity index. It appears that the impact of this condition is more significant for gestational hypertension and mild preeclampsia, having a lower impact as a risk factor for severe preeclampsia (Table II).

The 'tree' classification test on the impact of obesity as a risk factor for the studied groups shows the existence of a statistically significant difference depending on belonging to one of the three categories. Thus, obese patients have a higher risk of moderate preeclampsia, following gestational hypertension and finally severe preeclampsia (Fig. 3).

Through the binary logistic regression model, which has the category as dependent variable, the analysis of group 4, patient with mild preeclampsia was compared with group 5 , patients with severe preeclampsia regarding the predictive value of the significant risk factors obtained from the previous statistical analysis, considered as independent variables. Thus, we intended to highlight the predictive factors that significantly influence the development of severe preeclampsia in patients with moderate preeclampsia. The model has a $\chi^{2}(3)=15.9, \mathrm{P}<0.05$ and explains $26.4 \%$ of patients' probability of developing severe preeclampsia, when they have moderate preeclampsia (Nagelkerke $=0.264$ ). There are no large correlation factors between the independent variables, so the collinearity can be excluded (Table III).

According to the results presented in Table III, obesity seems to be a protective factor for patients with obesity and mild preeclampsia to develop severe preeclampsia, instead a cardiac pathological substrate or impaired hepatic function of patients with moderate preeclampsia is an important negative predictor, increasing the risk of developing severe preeclampsia. In order of impact, the cardiac pathological substrate prevails, followed by the associated liver pathology.

\section{Discussion}

In the study group, patients with mild preeclampsia predominated, the incidence during the 5 years of study was $2.8 \%$, with a $1.3 \%$ incidence of severe preeclampsia and $3.57 \%$ of these cases were complicated with eclampsia. According to the data reported in the literature, namely a declared incidence of pre-eclampsia of $2-8 \%$ with a progression rate to severe forms of $3.4-3.8 \%$ (6) our results corresponded with these data. The incidence of cesarean birth exceeded $90 \%$ in all the studied groups, and was $100 \%$ in the group of patients 


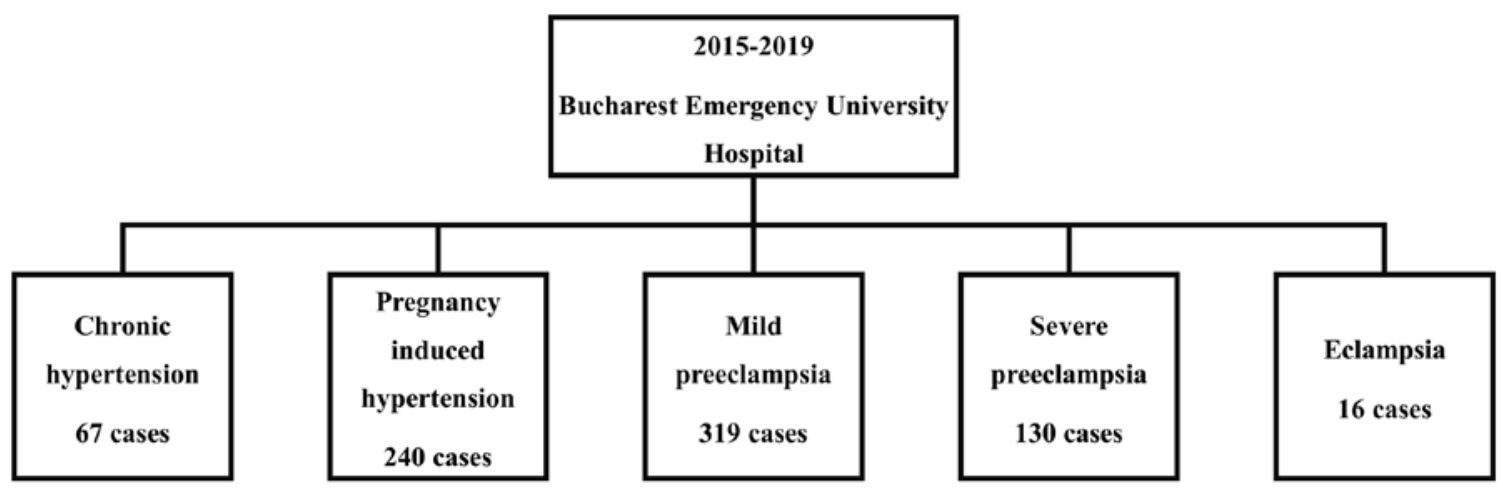

Figure 1. Summary of the database and the number of cases included in each of the five categories.
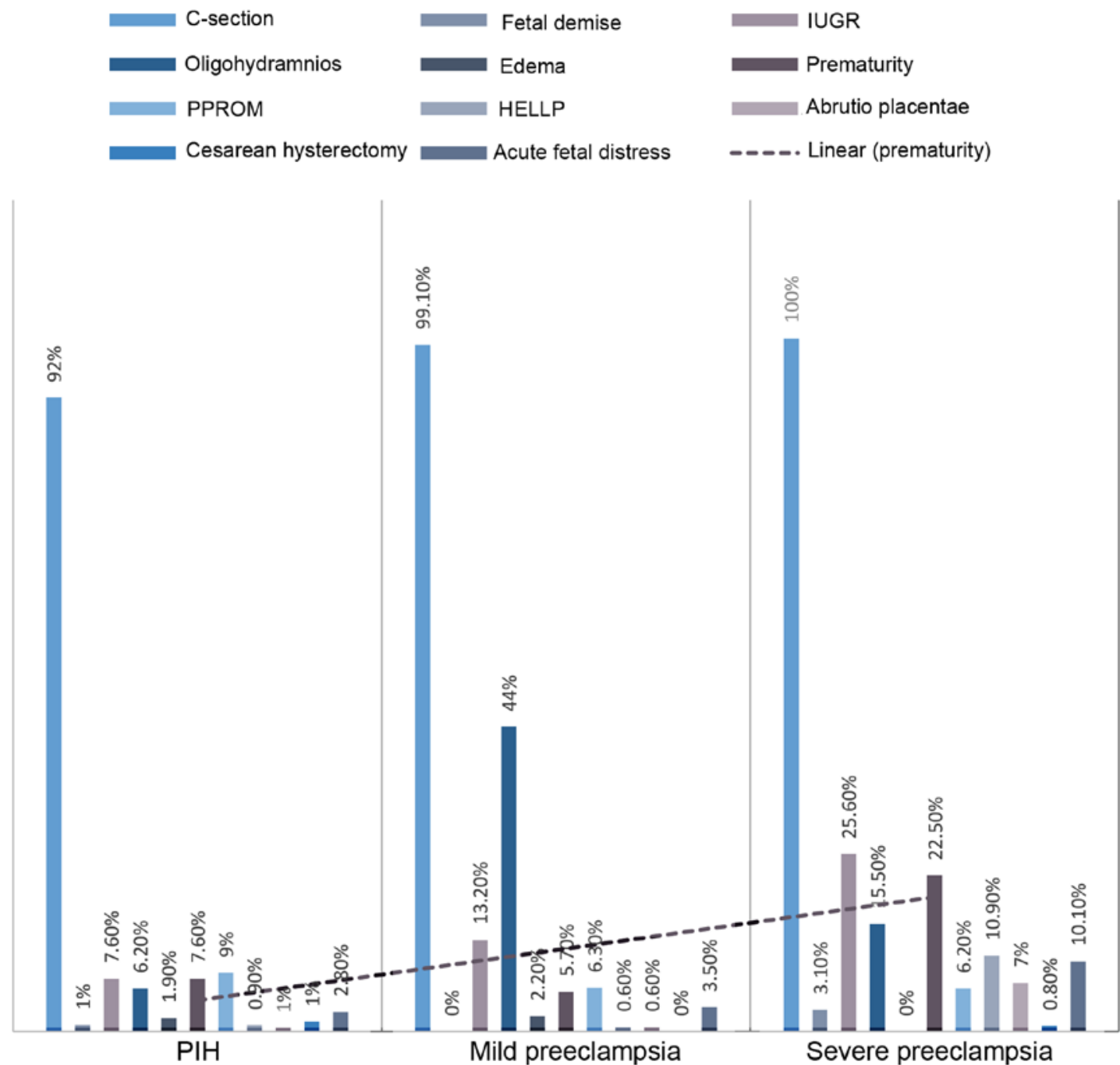

Figure 2. Complications associated with the study groups. PIH, pregnancy induced hypertension; IUGR, intrauterine growth restriction; PPROM, preterm premature rupture of membranes.

with eclampsia, which is justified by the urgent nature of these cases, the presentation in the stage of decompensation of a significant proportion of these cases and the need for an emergency termination of pregnancy, for both maternal and fetal purposes (7).

At first glance, through the graphical presentation of the study group, it can be observed that preterm birth predominates, with a significant incidence in severe preeclampsia. As noted in the results chapter, obesity is a dominant factor, present in $>50 \%$ of cases with mild preeclampsia.

Obesity is considered to be a mild risk factor for preeclampsia (8), the relationship between these two pathologies has long been studied in the context in which in the last quarter of century has experienced a constant upward trend 


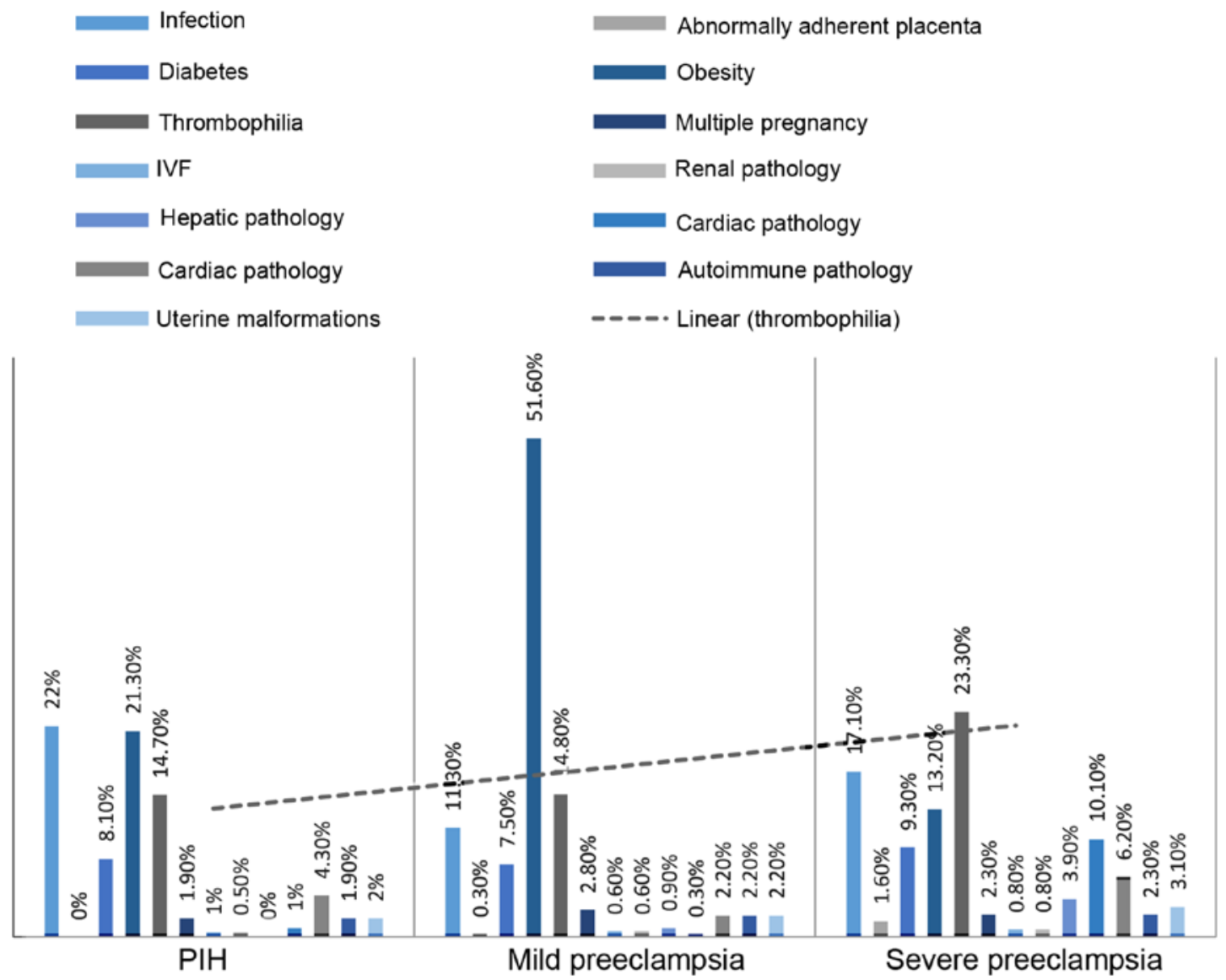

Figure 3. The studied groups and the main associated risk factors analyzed as well as the frequency for each study group for the purpose of the subsequent comparative analysis regarding the degree of association of each risk factor with the studied pathology. VF, in vitro fertilisation.

Table I. Risk factors and complications for the general population, in groups of women with gestational hypertension, moderate preeclampsia and severe preeclampsia.

\begin{tabular}{|c|c|c|c|c|c|c|c|c|c|c|c|c|}
\hline \multirow{2}{*}{$\begin{array}{l}\text { Factor/ } \\
\text { complication }\end{array}$} & \multicolumn{4}{|c|}{$\mathrm{PIH}$} & \multicolumn{4}{|c|}{ Mild preeclampsia } & \multicolumn{4}{|c|}{ Severe preeclampsia } \\
\hline & $\mathrm{U}$ & $\mathrm{z}$ & P-value & $\mathrm{r}$ & $\mathrm{U}$ & $\mathrm{z}$ & P-value & $\mathrm{r}$ & $\mathrm{U}$ & $\mathrm{z}$ & P-value & $\mathrm{r}$ \\
\hline Obesity & 8,961 & -2.3 & 0.02 & -0.13 & 9,449 & -7.16 & 0 & -0.35 & & & & \\
\hline Thrombophilia & & & & & 14,663 & -2 & 0.04 & -0.1 & -0.1 & -3.1 & 0 & -0.21 \\
\hline IUGR & & & & & 14,595 & -2.2 & 0.02 & -0.11 & & & & \\
\hline Oligohydramnios & & & & & 9,377 & -7.5 & 0 & -0.37 & $5,643.5$ & -3.1 & 0 & -0.21 \\
\hline Fetal demise & & & & & 15,264 & -3.5 & 0 & -0.17 & & & & \\
\hline Prematurity & & & & & & & & & 5,387 & -3.4 & 0 & -0.23 \\
\hline Acute fetal distress & & & & & & & & & 5,929 & -2.4 & 0.01 & -0.16 \\
\hline Abruptio placentae & & & & & & & & & $6,064.5$ & -2.2 & 0.03 & -0.15 \\
\hline
\end{tabular}

$r$, represents the size of the effect of the respective variable, that is, the greater the value of $r$ the more important the influence of the variable. IUGR, intrauterine growth restriction, PIH, pregnancy induced hypertension.

Table II. Frequency of obesity in study groups.

\begin{tabular}{lcc}
\hline Group & No (\%) & Yes (\%) \\
\hline Pregnancy induced hypertension & 77.7 & 22.3 \\
Mild preeclampsia & 48.4 & 51.6 \\
Severe preeclampsia & 86.8 & 13.2 \\
\hline
\end{tabular}

of both preeclampsia and obesity (9). In a previous study (10), the association of obesity and gestational hypertension with preeclampsia was investigated, and it was reported that the risk of developing a hypertensive disease in pregnancy increases proportionally to the increase of BMI.

We can conclude regarding the risk for a certain category of hypertensive disease of obese patients that obesity, as a 
Table III. Multinominal regression model for the group of patients with moderate preeclampsia compared with the group of patients with severe preeclampsia.

\begin{tabular}{|c|c|c|c|}
\hline \multirow[b]{2}{*}{ Factor } & \multicolumn{3}{|c|}{$95 \% \mathrm{CI}$ for $\operatorname{Exp}(\mathrm{B})$} \\
\hline & $\mathrm{B}(\mathrm{SE})$ & Inferior & Superior \\
\hline Obesity $^{\mathrm{a}}$ & 7.02 & 3.9 & 12.6 \\
\hline Thrombophilia & 1.12 & 0.62 & 2.03 \\
\hline Cardiac pathology ${ }^{a}$ & 0.035 & 0.004 & 0.293 \\
\hline Hepatic pathology & 0.12 & 0.023 & 0.604 \\
\hline Endocrine pathology & 0.359 & 0.113 & 1.14 \\
\hline
\end{tabular}

${ }^{\mathrm{a}} \mathrm{P}<0.05$.

risk factor, significantly impacts all categories of hypertension in pregnancy, from gestational hypertension to severe preeclampsia, but has an impact more significant as a risk factor for mild preeclampsia $(\mathrm{r}=7.16)$.

The mechanism by which obesity is an important risk factor for mild preeclampsia is dictated by the multiple common features of both obesity and preeclampsia, namely: hyperinsulinism, increased insulin resistance, increased leptin, TNF- $\alpha$, IL- 6 and quantitative C-reactive protein with altered lipid profile and decreased flow-mediated vasodilation (11). Both hyperinsulinism and increased insulin resistance are conditions that precede pre-eclampsia (12), all of which are among the most important associations of obesity. Regarding the role of adiponectins, leptin and proinflammatory cytokines in the pathophysiological mechanism of preeclampsia, adipose tissue has been shown to produce low levels of adiponectin with increased levels of proinflammatory cytokines, such as TNF- $\alpha$, and IL-6 leading to a proinflammatory status characterized by endothelial dysfunction and increased insulin resistance (13). In obese patients, there is an increased production of leptin, with a inflammatory effect and a low production of adiponectins with an anti-inflammatory effect resulting in a low degree systemic inflammation and increased insulin resistance, status that also characterizes preeclampsia, cardiovascular disease and type II diabetes (14). The importance of leptin in the development of preeclampsia is due to the fact that its high levels found in obesity, reduce the proliferation of the cytotrophoblast, a basic pathophysiological mechanism of preeclampsia, and increase the blood pressure and the level of placental factors (15).

Endothelial dysfunction is undoubtedly present in obese persons, being caused by the unbalanced production of metabolites of adipose tissue. The context characterized by an increased BMI, respectively, with increased leptin levels and other pro-inflammatory adiponectins, affects nicotinamide adenine dinucleotide phosphate (NADPH) oxidase, which influences endothelium-dependent vasodilation by increasing nitric oxide degradation (16). Nitric oxide deficiency affects the integrity and functioning of the endothelium, which in a pregnancy context, where the role of the endothelium is fundamental in the remodeling of uterine arteries and in hemodynamic adaptations, becoming a determining factor in the development of preeclampsia.

Nitric oxide (NO) results from the synthesis of the amino acid L-arginine in the presence of NO synthase, which is a family of enzymes dependent on NADPH and calcium. NO-endothelial synthase is expressed at the level of human placental syncytiotrophoblast and at the level of extravillous trophoblast, indicating that placental nitric oxide production plays an important role in the vascular adaptation required to ensure proper flow and avoid generalized peripheral vasoconstriction status (17), which undoubtedly characterizes preeclampsia.

Through the above mentioned arguments, an association link is clear between the existence of obesity, preeclampsia and the pathophysiological mechanisms involved. This remains a current problem, in the context in which the rate of obesity and preeclampsia in pregnancy has tripled in the recent decades and is constantly increasing and giving the fact that these pathologies associate a significant rate of maternal and fetal morbidity and mortality. The results obtained show that obesity is an important risk factor for mild preeclampsia, but not for severe preeclampsia.

\section{Acknowledgements}

Not applicable.

\section{Funding}

No funding was received.

\section{Availability of data and materials}

The datasets used and/or analyzed during the current study are available from the corresponding author on reasonable request.

\section{Authors' contributions}

DN, FG and NT contributed substantially to the statistical analysis of the data. FPP, ID and MMC collected, analyzed and interpreted the patient data regarding the impact of obesity on the evolution of a hypertensive pathology that complicates pregnancy. REB, CAZ and NT contributed substantially to the conception of the study and the data interpretation, and were major contributors in the writing of the manuscript. All authors read and approved the final version of the manuscript.

\section{Ethics approval and consent to participate}

Not applicable.

\section{Patient consent for publication}

Not applicable.

\section{Competing interests}

The authors declare that they have no competing interests. 


\section{References}

1. No authors listed: Report of the National High Blood Pressure Education Program Working Group on high blood pressure in pregnancy. Am J Obstet Gynecol 183: S1-S22, 2000.

2. Sibai BM and Stella CL: Diagnosis and management of atypical preeclampsia-eclampsia. Am J Obstet Gynecol 200: 481 e1-481.e7, 2009.

3. Thornton CE, Makris A, Ogle RF, Tooher JM and Hennessy A: Role of proteinuria in defining pre-eclampsia: Clinical outcomes for women and babies. Clin Exp Pharmacol Physiol 37: 466-470, 2010.

4. HomerCS, Brown MA, Mangos G and Davis GK: Non-proteinuric pre-eclampsia: A novel risk indicator in women with gestational hypertension. J Hypertens 26: 295-302, 2008.

5. Barton JR and Sibai BM: Diagnosis and management of hemolysis, elevated liver enzymes, and low platelets syndrome. Clin Perinatol 31: 807-833, vii, 2004.

6. Osmanağaoğlu MA, Erdoğan I, Zengin U and Bozkaya H: Comparison between HELLP syndrome, chronic hypertension, and superimposed preeclampsia on chronic hypertension without HELLP syndrome. J Perinat Med 32: 481-485, 2004.

7. Murray D, O'Riordan M, Geary M, Phillips R, Clarke T and McKenna P: The HELLP syndrome: Maternal and perinatal outcome. Ir Med J 94: 16-18, 2001.

8. Brown CE, Cunningham FG and Pritchard JA: Convulsions in hypertensive, proteinuric primiparas more than 24 hours after delivery. Eclampsia or some other cause? J Reprod Med 32: 499-503, 1987.

9. Zeeman GG: Neurologic complications of pre-eclampsia. Semin Perinatol 33: 166-172, 2009.

10. Belfort MA, Saade GR, Grunewald C, Dildy GA, Abedejos P, Herd JA and Nisell H: Association of cerebral perfusion pressure with headache in women with pre-eclampsia. Br J Obstet Gynaecol 106: 814-821, 1999.
11. Ghulmiyyah L and Sibai B: Maternal mortality from preeclampsia/eclampsia. Semin Perinatol 36: 56-59, 2012.

12. LeFevre ML; U.S. Preventive Services Task Force: Low-dose aspirin use for the prevention of morbidity and mortality from preeclampsia: U.S. Preventive Services Task Force recommendation statement. Ann Intern Med 161: 819-826, 2014.

13. Wang Y, Beydoun MA, Liang L, Caballero B and Kumanyika SK: Will all Americans become overweight or obese? estimating the progression and cost of the US obesity epidemic. Obesity (Silver Spring) 16: 2323-2330, 2008.

14. Yogev Y and Catalano PM: Pregnancy and obesity. Obstet Gynecol Clin North Am 36: 285-300, viii, 2009.

15. Sierra-Laguado J, García RG, Celedón J, Arenas-Mantilla M, Pradilla LP, Camacho PA and López-Jaramillo P: Determination of insulin resistance using the homeostatic model assessment (HOMA) and its relation with the risk of developing pregnancy-induced hypertension. Am J Hypertens 20: 437-442, 2007.

16. Lopez-Jaramillo P: The role of adiponectin in cardiometabolic diseases: Effects of nutritional interventions. J Nutr 146: 422S-426S, 2016.

17. Spradley FT, Palei AC and Granger JP: Increased risk for the development of preeclampsia in obese pregnancies: Weighing in on the mechanisms. Am J Physiol Regul Integr Comp Physiol 309: R1326-R1343, 2015.

(i) $($ This work is licensed under a Creative Commons Attribution-NonCommercial-NoDerivatives 4.0 International (CC BY-NC-ND 4.0) License. 